\title{
Fragmentation de Marangoni : les gouttes qui s'éclatent
}

Ludovic Keiser, Hadrien Bense, Cyril Sturtz, Pierre Colinet, Benoît Roman, José Bico et Étienne Reyssat (etienne.reyssat@espci.fr)

Laboratoire de Physique et Mécanique des Milieux Hétérogènes (PMMH), (CNRS UMR 7636, ESPCI Paris, PSL Research Univ., Univ. Paris Diderot), Sorbonne Université, 7 quai Saint-Bernard, barre Cassan, 75005 Paris

Une goutte d'eau déposée

à la surface d'un bain d'huile

de tournesol forme une lentille

liquide flottante. Une goutte

d'alcool, au contraire, s'étale

à la surface de l'huile. Un mélange

d'eau et d'alcool produit

un spectaculaire phénomène

d'étalement et de fragmentation

spontanée.

Si la goutte contient suffisamment

d'alcool, elle donne naissance

en quelques secondes

à une myriade de gouttelettes

dont la taille dépend fortement

de la composition initiale.

La combinaison

de l'hydrodynamique, du mouillage

et de l'évaporation est à l'origine

de cette instabilité originale.
Autour d'une table, une quinzaine de gamins, penchés sur une assiette remplie d'huile de cuisine, s'émerveillent devant un complexe ballet de gouttelettes colorées. C'est le genre de rassemblement qu'avait l'habitude de produire notre collègue et ami Gustavo Gutiérrez ${ }^{(a)}$, jovial bonhomme vénézuélien, pour faire découvrir la beauté des sciences aux enfants de Caracas. Nous avons eu la chance d'être nous aussi ces gamins, dans le milieu des années 2000, lorsque Gustavo a présenté cette expérience lors de la réunion hebdomadaire de notre laboratoire.

Une couche d'huile de tournesol d'environ $1 \mathrm{~cm}$ de profondeur est versée dans une assiette. Lorsqu'on dépose sur ce bain une goutte d'eau suffisamment petite, elle flotte à la surface et adopte la forme d'une lentille. Si on y dépose plutôt une goutte d'éthanol pur, celle-ci s'étale spontanément, puis s'évapore, restituant la surface d'huile immaculée. Que peut-il bien advenir lorsqu'on dépose un mélange d'eau et d'alcool (dont on discutera la composition plus loin), additionné d'un peu de colorant?

On observe qu'un mélange suffisamment riche en alcool commence par s'étaler. Le rayon de la goutte de mélange déposée sature toutefois après quelques secondes à une valeur de l'ordre de quelques centimètres. La frontière de la goutte mère se déstabilise et une myriade de gouttelettes est formée et expulsée vers l'extérieur, alimentant un magnifique motif de points colorés (fig. 1). Cette fragmentation spontanée est sensible à la profondeur du bain d'huile, à la concentration en alcool du mélange, ainsi qu'à la vitesse d'évaporation, comme on le montre très simplement en soufflant délicatement sur la goutte pendant l'expérience. Enfin, après une dizaine de secondes, la goutte mère se rétracte et laisse à la surface du bain un arrangement de gouttelettes qui mûrit lentement.

Ces motifs spectaculaires, bien qu'éphémères, peuvent être fixés simplement par des techniques d'impression inspirées du suminagashi, l'art japonais du papier marbré (voir encadré 1).

\section{Un seuil d'apparition}

Ce phénomène spectaculaire se produit si le mélange initial est suffisamment riche en alcool. Nous avons montré qu'il était nécessaire que le mélange soit mouillant sur l'huile, ce qui n'est pas le cas de l'eau pure [1]. Autrement dit, l'étalement est favorisé si l'énergie de surface de l'huile est plus élevée que la somme des énergies huile/mélange et mélange/air de l'interface complexe formée lors de l'étalement de la goutte. Des mesures des différentes tensions interfaciales en jeu en fonction du titre alcoolique du mélange montrent que ce dernier ne mouille l'huile que s'il contient plus de 65\% d'éthanol. La concentration en alcool doit donc être plus élevée pour observer l'effet.

\section{Limite d'étalement}

Au-delà de ce seuil de concentration, la goutte commence à s'étaler puis s'arrête. Une odeur légèrement éthérée nous indique qu'une partie de l'alcool s'est évaporée. Ce dernier est en effet bien plus volatil que l'eau, qui ne s'évapore pratiquement pas à l'échelle de temps de notre expérience (quelques dizaines de secondes). La goutte appauvrie en alcool, ne mouille plus l'huile et tend à se rétracter, mais pourquoi ne revient-elle pas à un état de lentille ? 


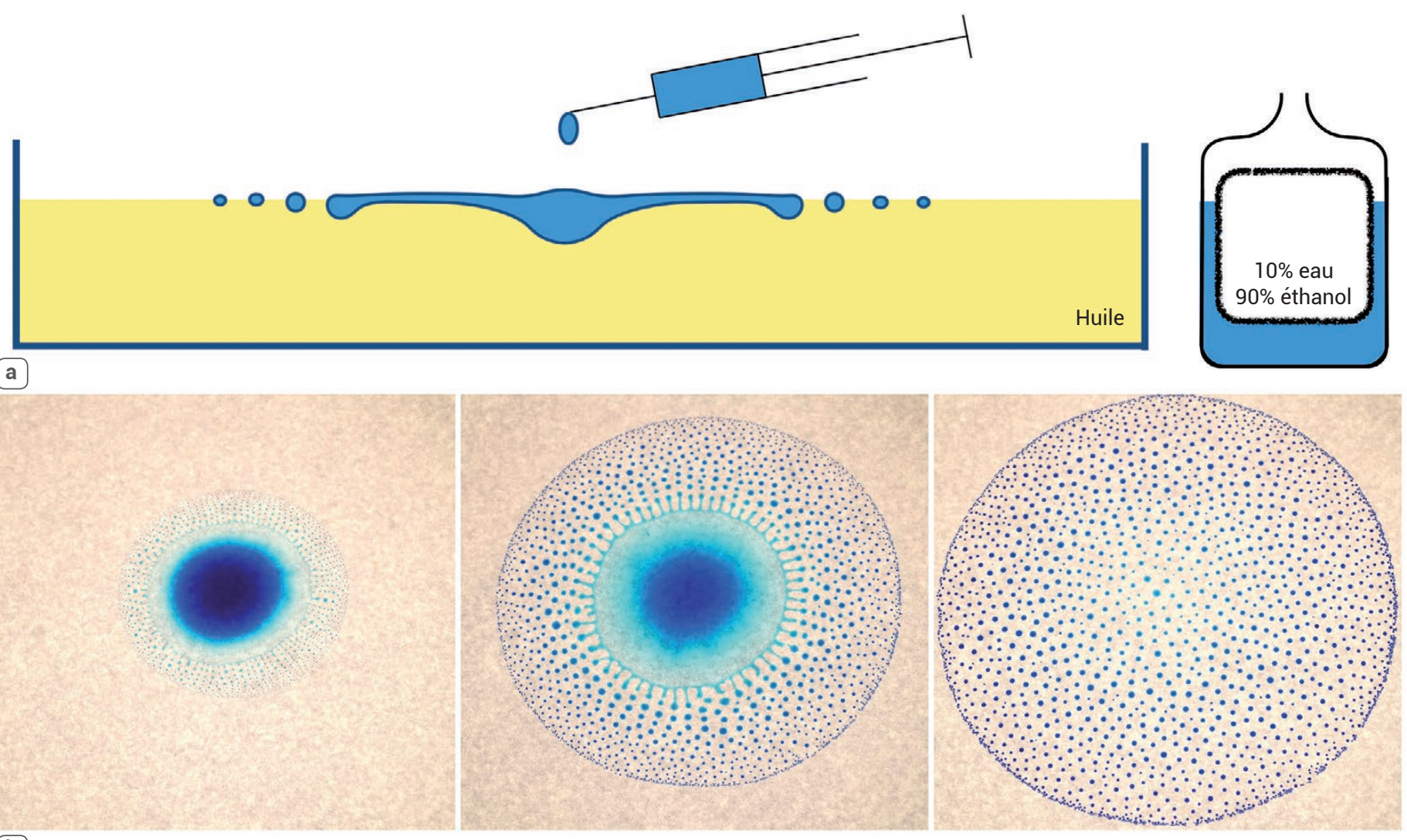

(b)

1. Expérience d'éclatement d'une goutte.

(a) Dispositif expérimental : on dépose sur un bain d'huile de tournesol une goutte de mélange d'eau et d'alcool.

(b) Si le mélange est suffisamment riche en alcool, la goutte s'étale et se fragmente spontanément. En quelques secondes, elle donne naissance à une myriade de gouttelettes.

\section{Impressions artistiques}

Pour ajouter une dernière touche au « ouaah! » engendré par un spectaculaire éclatement, voyons comment capturer ces motifs éphémères. En déposant, au moment voulu, une feuille de papier sur le bain, il est possible de fixer le moucheté coloré de gouttelettes formé à la surface (fig. E1). Cette technique est un avatar de celle que les Japonais nomment "suminagashi". Cet art des encres qui flottent sur l'eau permet de produire des motifs de cernes très élaborés qui sont ensuite transférés sur papier.

Vous aurez peut-être déjà remarqué les revers de couverture marbrés ornant certains ouvrages anciens, imprimés selon des principes similaires. Cette technique a été récemment reprise par l'artiste Xavier Martinez [2]. Voilà un moyen simple de fixer des merveilles passagères (fig. E2). À vous de jouer [3] !

E1. Un motif de gouttelettes en cours de formation est capturé sur un papier déposé à la surface du bain.

E2. "Sous-marin », par Xavier Martinez [2]. Des cernes d'encre de suminagashi sont déformés par le passage d'un sous-marin fantomatique à la surface du bain.
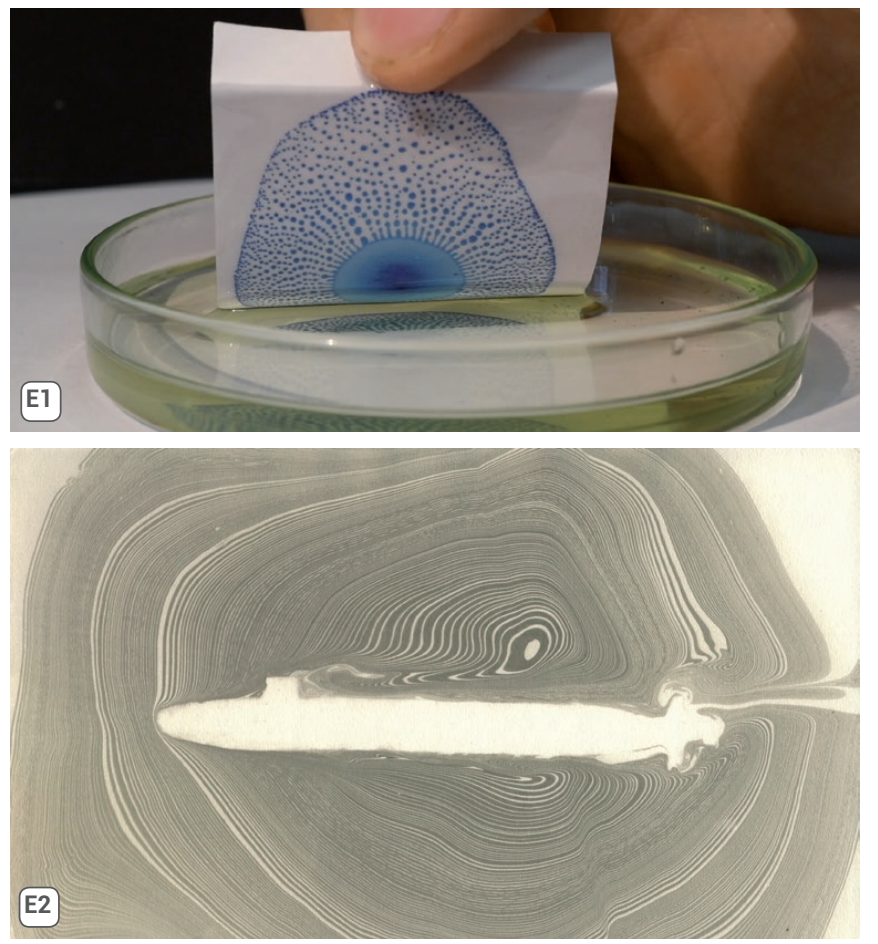
\〉

\section{Une goutte en forme d'œuf au plat}

Observons la goutte plus attentivement. La couleur de son centre est plus prononcée que dans les régions périphériques (fig. 2a). Ceci traduit le fait que la goutte est plus épaisse au centre et amincie au bord. Des mesures quantitatives du profil d'épaisseur des gouttes peuvent aussi être réalisées par interférométrie, en utilisant les couleurs qu'on peut parfois observer sur les taches d'huile étalées sur une route mouillée (fig. 2b). Au centre de la goutte, l'épaisseur de mélange est de l'ordre d'une fraction de millimètre. À la périphérie, le film mesure parfois moins d'un micromètre !

\section{Un gradient de concentration}

À taux d'évaporation égal, l'effet de l'évaporation est plus sensible dans les régions minces en périphérie de la goutte qui s'appauvrissent plus rapidement en alcool. Cette inhomogénéité de concentration se traduit aussi par l'apparition d'un gradient de tension interfaciale dirigé selon le rayon de la goutte. Ce gradient induit des contraintes mécaniques qui se développent le long de la surface, et drainent le liquide du centre de la goutte vers la périphérie (fig. 3). Cet écoulement, facilité par la présence du bain d'huile, s'oppose ainsi au retrait du bord de la goutte. Du liquide tend donc à s'accumuler en bord de goutte, formant ainsi une surépaisseur. C'est ce bourrelet qui se déstabilise et se fragmente, éjectant jusqu'à un milliard de microgouttes en quelques secondes ! Ce tore liquide, à l'instar de tout filament liquide, est en effet instable du point de vue de la tension superficielle : comme le filet d'eau qui s'écoule de votre robinet, il se fragmente en une multitude de gouttes, dont la surface totale est inférieure à celle du cylindre de même volume. Vous aurez sans doute remarqué qu'un jet de petit diamètre se fragmente en petites gouttes: la taille caractéristique des gouttes est proportionnelle au diamètre du jet. Dans l'expérience qui nous intéresse ici, la taille des gouttelettes reflète l'épaisseur du mince bourrelet qui se forme à la périphérie de la goutte.
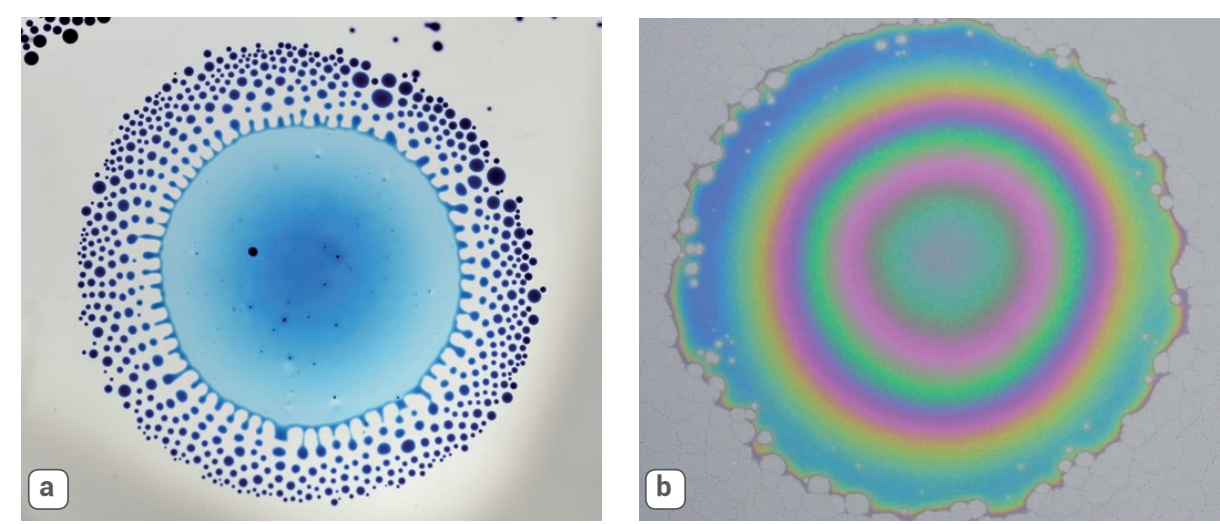

2. Profil d'épaisseur de la goutte.

(a) Une goutte de liquide chargée d'un peu de colorant apparaît foncée au centre, où elle est plus épaisse. En périphérie, la couleur moins dense traduit la faible épaisseur du film. Cette méthode est adaptée pour des films de mélange suffisamment épais (10 à $1000 \mu \mathrm{m}$ typiquement), c'est-à-dire pour un mélange dont la concentration en alcool est légèrement au-dessus du seuil d'observation du phénomène.

(b) Pour des gouttes suffisamment minces (100 nm à quelques $\mu \mathrm{m})$, c'est-à-dire pour les mélanges les plus riches en alcool, des mesures quantitatives de l'épaisseur peuvent être réalisées par interférométrie.

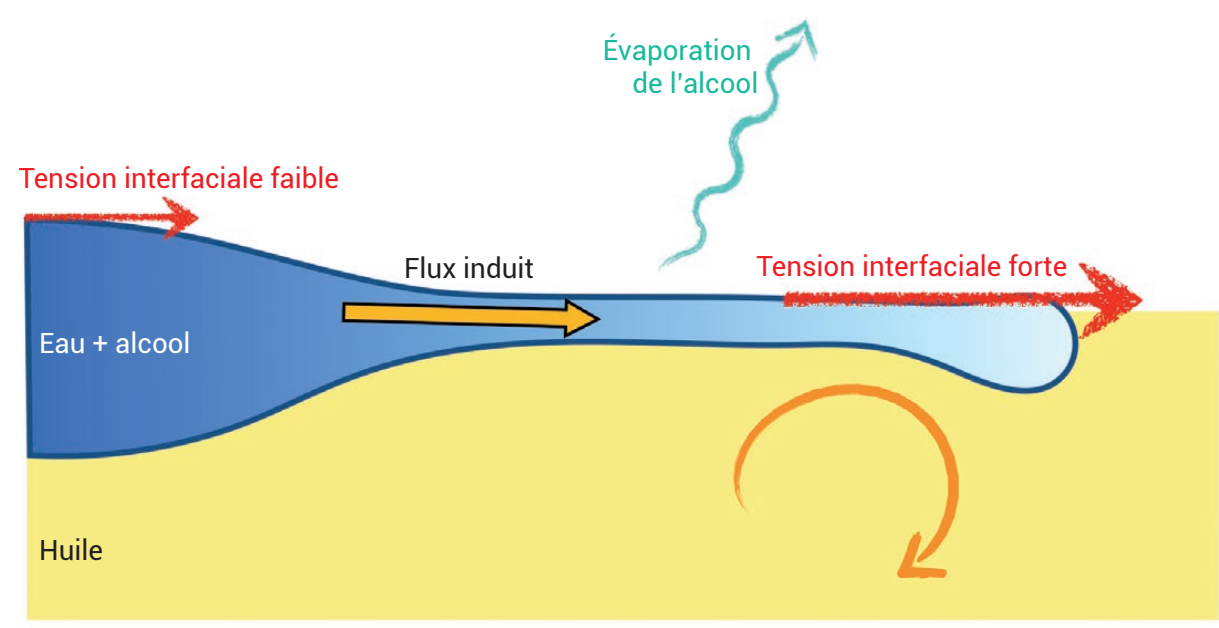

3. L'étalement de Marangoni. Sous l'effet de l'évaporation, la périphérie amincie de la goutte s'appauvrit en alcool. L'inhomogénéité de composition se traduit par un déséquilibre de tension interfaciale qui draine le mélange du centre vers la périphérie de la goutte où le liquide s'accumule dans un bourrelet. Les écoulements dans l'huile sous la goutte (flèche orange) accompagnent cet étalement de Marangoni.

\section{Références}

1• L. Keiser et al., Phys. Rev. Lett., 118 (2017) 074504.

2. www.xaviermartinez.eu

3. www.youtube.com/watch?v=7-HpbBnfIK4

4 J. B. Fournier et A.-M. Cazabat, Europhys. Lett., 20 (1992) 517.

5• M. Roché et al., Phys. Rev. Lett. 112 (2014) 208302.

6• F. Wodlei et al., Nature Comm., 9 (2018) 820.

$7 \cdot G$. Durey, projet Lutétium, www.youtube.com/watch?v=h3iUy4Wg8lg\&t=61s 


\section{Gradients de tension de surface : l'effet Marangoni}

Vous pourrez retrouver dans un verre de vin un autre avatar de ce phénomène spectaculaire : le film déposé en tournant délicatement le verre forme un bourrelet qui se déstabilise en "larmes » ou " jambes » appréciées des œnologues [4]. Là encore, l'évaporation préférentielle de l'alcool sur la partie supérieure du film se traduit par des gradients de tension superficielle qui drainent le film liquide vers le haut du verre, formant ainsi le bourrelet. En grossissant, ce renflement devient sensible à la gravité, et libère des gouttes qui retombent dans le verre. De taille millimétrique, ces gouttes sont sensiblement plus grosses que celles de l'expérience décrite précédemment. Il s'agit ici d'une variante de l'instabilité hydrodynamique de RayleighTaylor, qui donne naissance à des gouttes sous un plafond humide.

Les écoulements engendrés par des gradients de concentration sont qualifiés "d'effets Marangoni » en l'honneur du physicien italien qui s'est intéressé en 1865 à l'étalement d'un liquide sur un autre. Ces effets se manifestent également dans d'autres expériences de laboratoire mettant en jeu des ingrédients physiques similaires. Le dépôt d'une solution de surfactants à la surface de l'eau engendre ainsi des écoulements dans une région limitée par la dissolution des surfactants dans l'eau [5]. Une goutte de dichlorométhane déposée sur l'eau en présence de tensio-actifs donne aussi lieu à des phénomènes d'oscillations et de fragmentation spontanée en lien avec les observations discutées ici [6]. Il s'agit également d'une problématique industrielle importante. Les effets Marangoni peuvent en effet nuire à la qualité d'un revêtement en induisant une rugosité de surface en " peau d'orange ». Ce défaut, connu des peintres en bâtiment, apparait lors de l'application d'une couche de peinture trop épaisse sur une surface : au séchage, le rendu prend un aspect granuleux.

Le couplage des mécanismes physiques élémentaires de mouillage, lubrification, évaporation, fragmentation donne ainsi lieu à une classe de phénomènes riches, complexes mais aussi esthétiques [7], qu'on retrouve à la table d'un bon dîner comme au laboratoire ou dans de nombreux procédés industriels. 